\title{
ON CERTAIN ALGEBRAS OF ANALYTIC FUNCTIONS ON RIEMANN SURFACES
}

\author{
PENTTI JÄRVI
}

\section{Introduction}

Let $W$ be an open Riemann surface, and let $\beta$ denote the Kerékjártó-Stoillow ideal boundary of $W$. Denote by $A C(W)$ the class of analytic functions on $W$ which have a finite limit at each element of $\beta$, and denote by $M C(W)$ the class of meromorphic functions on $W$ which have a finite or infinite limit at each element of $\beta$. Whenever $f$ is a function of class $A C(W)$ or $M C(W)$, we let $f^{*}$ denote the extension of $f$ to the ideal boundary.

It is clear that $A C(W)$ can be regarded as a uniformly closed subalgebra of $C(W \cup \beta)$, the Banach algebra of continuous complex-valued functions on $W \cup \beta$. Of course, it may happen that $A C(W)$ reduces to the class of constants; this is the case e.g. if some element of $\beta$ admits realization as an isolated nondegenerate continuum or if the number of boundary elements is countable. It is also possible that $A C(W)$ fails to separate the points of $W$ (or $W \cup \beta$ ); see the example in Section 5. However, assuming that $W$ is a Riemann surface of finite genus and satisfies the reasonable condition

(*) $\quad M C(W)$ contains a function of bounded valence,

$A C(W)$ turns out to be a uniform algebra (for the definition see e.g. [3, p. 25]), provided $\beta$ contains essential elements in the sense to be defined in Section 4. This is the case to which we pay our attention in the present paper. In particular, we wish to discuss the relation between homomorphisms of the algebras $A C(W)$ and analytic mappings of the Riemann surfaces $W$. The considerations rely on an appropriate representation of the Riemann surfaces in question (Section 3), on a classification of boundary elements (Section 4) and, above all, on Arens' description of the maximal ideal spaces of certain function algebras ([2]). 


\section{Preliminaries}

Let $E$ be a proper closed subset of the Riemann sphere $\hat{C}=C \cup\{\infty\}$. Then $E$ is said to be of class $N_{C}$ if, for every domain $G \subset \hat{C}$ with $E \subset G$, every function $G \rightarrow C$, continuous on $G$ and analytic on $G \backslash E$, is actually analytic on $G$. The subclass of $N_{C}$ constituted by the totally disconnected members of $N_{C}$ is denoted by $N_{C}^{\prime}$. It is known that closed sets in $\hat{\boldsymbol{C}}$ of $\sigma$-finite linear measure are of class $N_{C}$ and, on the other hand, no set whose Hausdorff dimension exceeds 1 is of class $N_{C}$ ([4, Chapter 3]).

Given any Riemann surface $W$, a closed totally disconnected subset $E$ of $W$ is said to be of class $N_{C}^{\prime}$ in $W$ if for every parametric disc $(V, \varphi)$ of $W$ the compact parts of $\varphi(E \cap V) \subset \hat{C}$ are of class $N_{C}^{\prime}$. We shall need the following result from [5] ([5, Lemma 2]).

Lemma 1. Let $W$ be a Riemann surface, and let $f: W \rightarrow \hat{C}$ be a nonconstant analytic mapping.

(1) If $E \subset \hat{C}$ is of class $N_{C}^{\prime}$, then $f^{-1}(E)$ is of class $N_{C}^{\prime}$ in $W$.

(2) If $E$ is a compact set of class $N_{C}^{\prime}$ in $W$, then $f(E)$ is of class $N_{C}^{\prime}$.

We shall also make essential use of the following three lemmas; the proofs of Lemma 2 and Lemma 3 can be found in [5] and that of Lemma 4 in [12, p. 336] or in $[13$, p. 50$]$.

Lemma 2. Let $W$ be an open Riemann surface, let $\beta$ be the ideal boundary of $W$ and let $f \in A C(W)$. Then $f^{*}(\beta)=f^{*}(W \cup \beta)$. Moreover, if $f \in A C(W)$ is nonconstant, then the set

$$
\left\{z \in \hat{\boldsymbol{C}} \mid v_{f}(z)=\sum_{f(p)=z} n(p ; f)=\infty\right\}
$$

where $n(p ; f)$ denotes the multiplicity of $f$ at $p$, is residual in $f^{*}(\beta)$.

Lemma 3. Let $W$ be a compact Riemann surface, and let $E$ be a closed totally disconnected subset of $W$ failing to be of class $N_{C}^{\prime}$ in $W$. Then the class $A C(W \backslash E)$ contains a nonconstant function.

Lemma 4. Let $E$ be a closed nowhere dense subset of a Riemann surface W. Let $f: W \rightarrow \hat{\boldsymbol{C}}$ be a continuous light mapping such that $f$ is interior and locally sense-preserving on $W \backslash E$. Suppose that $f(E)$ contains no open set. Then $f$ is interior on $W$. 


\section{A representation theorem}

Let $W$ be an open Riemann surface of finite genus. It is well known that $W$ can be taken as a subregion of a compact Riemann surface $W^{*}$. If $W^{*} \backslash W$ is totally disconnected, we can identify $W^{*} \backslash W$ with $\beta$, the ideal boundary of $W$ (cf. Lemma 3). So in this case $W \cup \beta$ can be realized as a Riemann surface. We will show that the condition (*) of Section 1 guarantees this state of affairs.

Theorem 1. Let $W$ be an open Riemann surface of finite genus which satisfies the condition (*). Then there exist a compact Riemann surface $W^{*}$ and a closed totally disconnected set $E \subset W^{*}$ such that $W$ is conformally equivalent to $W^{*} \backslash E$.

Proof. Let $f$ be a function of class $M C(W)$ and of bounded valence, and let $f^{*}$ denote the extension of $f$ to $W \cup \beta$. Suppose that $\left(V_{n}\right)$ is a standard exhaustion of $W$, and denote by $F_{n}$ the closed set $f^{*}(\beta) \backslash f\left(W \backslash \bar{V}_{n}\right), n=1,2, \ldots$. By continuity, $F_{n}$ is a nowhere dense subset of $\hat{\boldsymbol{C}}$ for each $n$. Since $f$ has bounded valence, $f^{*}(\beta)$ is contained in $\bigcup_{n=1}^{\infty} F_{n}$. We conclude by Baire's theorem that $f^{*}(\beta)$ is nowhere dense in $\hat{\boldsymbol{C}}$.

By [8, Theorem 3], there exist a compact Riemann surface $S$, a closed totally disconnected set $F \subset S$ and a sense-preserving homeomorphism $\varphi: W \rightarrow S \backslash F$. It is readily seen that $\varphi$ admits a homeomorphic extension $\varphi^{*}: W \cup \beta \rightarrow S$. Consider the continuous mapping

$$
g=f^{*} \circ \varphi^{*-1}: S \rightarrow \hat{C} .
$$

It follows from the total disconnectedness of $F$ that $g$ is light. Moreover, $g \mid S \backslash F$ is interior and sense-preserving, and $g(F)=f^{*}(\beta)$ is nowhere dense, so that Lemma 4 applies. We infer that $g$ is interior on $S$.

By Stoillow's theorem ([11, p. 121]), there exist a compact Riemann surface $W^{*}$, a sense-preserving homeomorphism $\psi: S \rightarrow W^{*}$, and an analytic mapping $h: W^{*} \rightarrow \hat{\boldsymbol{C}}$ such that $g=h \circ \psi$. Let $E$ denote the totally disconnected set $\psi(F)$. We claim that $\psi \circ \varphi$ is a conformal homeomorphism $W \rightarrow W^{*} \backslash E$. Denote by $B_{f}$ the discrete set of zeros of the derivative of $f$. Let $p \in W \backslash B_{f}$ and select a neighborhood $U_{p}$ of $p$ such that $f \mid U_{p}$ is injective. Then we have

$$
\varphi \mid U_{p}=\left(g^{-1} \mid f\left(U_{p}\right)\right) \circ\left(f \mid U_{p}\right),
$$

where the branch of $g^{-1}$ is chosen in such a way that $g^{-1}(f(p))=\varphi(p)$. Similarly,

$$
\psi \mid \varphi\left(U_{p}\right)=\left(h^{-1} \mid f\left(U_{p}\right)\right) \circ\left(g \mid \varphi\left(U_{p}\right)\right),
$$

where the branch of $h^{-1}$ is chosen to satisfy $h^{-1}(g(\varphi(p))=\psi(\varphi(p))$. Thus

$$
(\psi \circ \varphi) \mid U_{p}=\left(h^{-1} \mid f\left(U_{p}\right)\right) \circ\left(f \mid U_{p}\right) .
$$

We infer that $\psi \circ \varphi$ is analytic on $W \backslash B_{f}$. Moreover, continuity and the discreteness of $B_{f}$ imply that $\psi \circ \varphi$ is analytic all over $W$. 
We will emphasize a certain special case in the following

Corollary. Let $W$ be an open Riemann surface of finite genus, and let $E$ be a totally disconnected subset of $\hat{\boldsymbol{C}}$. Suppose that $f: W \rightarrow \hat{\boldsymbol{C}} \backslash E$ is a proper analytic mapping. Then there exist a compact Riemann surface $W^{*}$ and a closed totally disconnected set $F \subset W^{*}$ such that $W$ is conformally equivalent to $W^{*} \backslash F$.

Proof. Since $f$ is proper, $\mathrm{Cl}(f ; \beta)$, the cluster set of $f$ attached to the ideal boundary $\beta$, is contained in $E$. By the total disconnectedness of $E, f$ admits a continuous extension $f^{*}$ to $W \cup \beta$ such that $f^{*}(\beta) \subset E$. It is readily seen that $v_{f}$ is finite and constant on $\hat{\boldsymbol{C}} \backslash E$. The assertion follows.

Remark. Suppose that $W$ is a Riemann surface which satisfies the hypotheses of Corollary, and let $W^{*}$ be a compact Riemann surface such that $W$ can be identified with a subregion of $W^{*}$. Then, in general, the set $F=W^{*} \backslash W$ need not be totally disconnected. In fact, it follows from [6, Theorem 1] and [10, Theorem X. 3 C] that all realizations of $W^{*} \backslash W$ are totally disconnected if and only if $E \in N_{S B}$ (for the definition and basic properties of the class $N_{S B}$, see [1]).

\section{Essential boundary elements}

Again let $W$ be a Riemann surface of finite genus satisfying the condition (*), and let $W^{*}$ and $E$ be as in Theorem 1. It is clear that the algebra $A C(W)$ can be identified with $A C\left(W^{*} \backslash E, W^{*}\right)$, the Banach algebra of complex-valued functions, defined and continuous on $W^{*}$ and analytic on $W^{*} \backslash E$. Suppose that $A C(W)$ is nontrivial, i.e. $A C(W)$ contains a nonconstant function. Then it follows from [2, Theorem 5.4] that $A C(W)$ separates the points of $W$ (even those of $W \cup \beta$ ). Actually, by the main result of [7], one can find, whatever the genus of $W$ may be, four functions in $A C(W)$ which separate the points of $W$. Consequently, $A C(W)$ is a uniform algebra.

Assume now that $A C(W)$ is nontrivial. Denote by $\partial A C(W)$ the Shilov boundary of $A C(W)$ (for the definition see [3, p. 10 and 25]). It is clear that $\partial A C(W) \subset \beta$. We say that a boundary element $p \in \beta$ is removable if $p \in \beta \backslash \partial A C(W)$, and $p \in \beta$ is said to be essential if $p \in \partial A C(W)$. The following theorem provides several useful characterizations of the removability of boundary elements.

Theorem 2. Let $W$ be a Riemann surface of finite genus which satisfies the condition (*), and suppose that $A C(W)$ is nontrivial. Let $p \in \beta$. Then the following conditions are equivalent:

(1) $p$ is removable.

(2) There is a neighborhood $U_{p} \subset W \cup \beta$ of $p$ with $\partial U_{p}$ in $W$ such that $f^{*}\left(\beta \cap U_{p}\right)$ is of class $N_{C}$ for some nonconstant $f \in M C(W)$. 
(3) Let $W^{*}$ and $E$ be as in Theorem 1, and let $\varphi$ denote a conformal mapping of $W$ onto $W^{*} \backslash E$ with a homeomorphic extension $\varphi^{*}: W \cup \beta \rightarrow W^{*}$. Then there is a neighborhood $U_{p} \subset W \cup \beta$ of $p$ with $\partial U_{p}$ in $W$ such that $\varphi^{*}\left(\beta \cap U_{p}\right)$ is of class $N_{C}^{\prime}$ in $W^{*}$.

(4) There is a neighborhood $U_{p} \subset W \cup \beta$ of $p$ with $\partial U_{p}$ in $W$ such that $f^{*}\left(\beta \cap U_{p}\right)$ is nowhere dense in $C$ for every $f \in A C(W)$.

Proof: $(1) \Rightarrow(3)$ : Suppose that $p_{0} \in \beta \backslash \partial A C(W)$. Since $\partial A C(W)$ is closed, there exists a neighborhood $U_{p_{0}} \subset W \cup \beta$ of $p_{0}$ such that $U_{p_{0}} \cap \partial A C(W)=\emptyset$ and $\partial U_{p_{0}} \subset W$. Assume that $\varphi^{*}\left(\beta \cap U_{p_{0}}\right)$ fails to be of class $N_{C}^{\prime}$ in $W^{*}$. Then, by Lemma 3 , there is a nonconstant function $f$ in $A C\left(W^{*} \backslash \varphi^{*}\left(\beta \cap U_{p_{0}}\right)\right)$. By the maximum principle,

Hence

$$
|f(p)|<\max \left\{\left|f^{*}(q)\right| \mid q \in W^{*}\right\} \text { for every } p \in W^{*} \backslash \varphi^{*}\left(\beta \cap U_{p_{0}}\right) .
$$

$$
\left|f\left(\varphi^{*}(p)\right)\right|<\max \left\{\left|f^{*}\left(\varphi^{*}(q)\right)\right| \mid q \in W \cup \beta\right\}
$$

for every $p \in(W \cup \beta) \backslash\left(\beta \cap U_{p_{0}}\right)$. This contradicts the relation $U_{p_{0}} \cap \partial A C(W)=\emptyset$. Thus $\varphi^{*}\left(\beta \cap U_{p_{0}}\right)$ is of class $N_{C}^{\prime}$ in $W^{*}$.

$(3) \Rightarrow(1)$ : Let $f \in A C(W)$. Since $\varphi^{*}\left(\beta \cap U_{p}\right)$ is of class $N_{C}^{\prime}$ in $W^{*}, f \circ \varphi^{*-1}$ is analytic on $\varphi^{*}\left(U_{p}\right)$. Therefore i.e.,

$$
\max \left\{\left|\left(f^{*} \circ \varphi^{*-1}\right)(q)\right| \mid q \in W^{*}\right\}=\max \left\{\left|\left(f^{*} \circ \varphi^{*-1}\right)(q)\right| \mid q \in E \backslash \varphi^{*}\left(U_{p}\right)\right\},
$$

$$
\max \left\{\left|f^{*}(q)\right| \mid q \in W \cup \beta\right\}=\max \left\{\left|f^{*}(q)\right| \mid q \in \beta \backslash U_{p}\right\} .
$$

Consequently, $p \in \beta \backslash \partial A C(W)$.

$(3) \Rightarrow(2)$ : Suppose that $f \in M C(W)$ is nonconstant. Because $f^{*}\left(\beta \cap U_{p}\right)=$ $\left(f^{*} \circ \varphi^{*-1}\right)\left(\varphi^{*}\left(\beta \cap U_{p}\right)\right)$, the assertion follows immediately from Lemma 1 . So we have even $f^{*}\left(\beta \cap U_{p}\right) \in N_{C}^{\prime}$.

$(2) \Rightarrow(3)$ : Let $f \in M C(W)$, and assume that $f^{*}\left(\beta \cap U_{p}\right)=\left(f^{*} \circ \varphi^{*-1}\right)\left(\varphi^{*}\left(\beta \cap U_{p}\right)\right)$ is of class $N_{C}$ for some neighborhood $U_{p} \subset W \cup \beta$ of $p$ with $\partial U_{p}$ in $W$. Then, by Lemma $4,\left(f^{*} \circ \varphi^{*-1}\right) \mid \varphi^{*}\left(U_{p}\right)$ is interior. By Stoïlow's theorem, $\left(f^{*} \circ \varphi^{*-1}\right) \mid \varphi^{*}\left(U_{p}\right)$ is a local homeomorphism off a discrete set in $\varphi^{*}\left(U_{p}\right)$. We infer that $f^{*}\left(\beta \cap U_{p}\right)$ is actually of class $N_{C}^{\prime}$. We can now readily see, appealing to the local invertibility of $f^{*} \circ \varphi^{*-1}$, that $f^{*} \circ \varphi^{*-1}$ is analytic on $\varphi^{*}\left(U_{p}\right)$. Hence, by Lemma $1, \varphi^{*}\left(\beta \cap U_{p}\right)$ is of class $N_{C}^{\prime}$ in $W^{*}$.

(3) $\Leftrightarrow(4)$ : Suppose that $\varphi^{*}\left(\beta \cap U_{p}\right)$ is of class $N_{C}^{\prime}$ in $W^{*}$, and let $f \in A C(W)$. Since $f^{*} \circ \varphi^{*-1}$ is analytic on $\varphi^{*}\left(U_{p}\right), f^{*}\left(\beta \cap U_{p}\right)=\left(f^{*} \circ \varphi^{*-1}\right)\left(\varphi^{*}\left(\beta \cap U_{p}\right)\right)$ is totally disconnected. Suppose conversely that $\varphi^{*}\left(\beta \cap U_{p}\right)$ fails to be of class $N_{C}^{\prime}$ in $W^{*}$. By Lemma 3 there exists a nonconstant function $g$ in $A C\left(W^{*} \backslash \varphi^{*}\left(\beta \cap U_{p}\right)\right)$. By Lemma $2 g^{*}\left(\varphi^{*}\left(\beta \cap U_{p}\right)\right)$ contains interior points. Since $f=g^{*} \circ \varphi$ belongs to $A C(W)$, the proof is herewith complete.

Remark. It follows from Lemma 3 that $A C(W)$ is nontrivial if and only if $E$ fails to be of class $N_{C}^{\prime}$ in $W^{*}$. 


\section{Main results}

Let $W$ and $W^{\prime}$ be open Riemann surfaces with ideal boundaries $\beta$ and $\beta^{\prime}$, respectively. Suppose that $\varphi$ is an analytic mapping $W \rightarrow W^{\prime}$ which admits a continuous extension $\varphi^{*}: W \cup \beta \rightarrow W^{\prime} \cup \beta^{\prime}$. Then, clearly, $f \mapsto f \circ \varphi$ is an algebra homomorphism of $A C\left(W^{\prime}\right)$ into $A C(W)$, and the homomorphism is injective whenever $\varphi$ is nonconstant. Conversely we have

Theorem 3. Let $W$ and $W^{\prime}$ be open Riemann surfaces with ideal boundaries $\beta$ and $\beta^{\prime}$, respectively. Suppose that $W^{\prime}$ is of finite genus and satisfies the condition (*); suppose further that $\beta^{\prime}$ contains no removable element. Let $T$ be an algebra homomorphism of $A C\left(W^{\prime}\right)$ into $A C(W)$ such that the dimension of its range exceeds 1 . Then there exists a unique nonconstant analytic mapping $\psi$ of $W$ into $W^{\prime}$, admitting a continuous extension $\psi^{*}: W \cup \beta \rightarrow W^{\prime} \cup \beta^{\prime}$, such that $T f=f \circ \psi$ for every $f \in A C\left(W^{\prime}\right)$. In particular, in case that $W$, too, fulfils the conditions imposed before on $W^{\prime}, W$ and $W^{\prime}$ are conformally equivalent if and only if $A C(W)$ and $A C\left(W^{\prime}\right)$ are algebraically. isomorphic.

Proof. Denote by $W^{\prime *}$ or $E^{\prime}$ the compact Riemann surface or the closed totally disconnected subset of $W^{\prime *}$, respectively, described in Theorem 1. Similarly, let $\varphi^{\prime}$ denote a conformal mapping of $W^{\prime}$ onto $W^{\prime *} \backslash E^{\prime}$ with a homeomorphic extension $\varphi^{\prime *}: W^{\prime} \cup \beta^{\prime} \rightarrow W^{\prime *}$. As in Section 4, we may identify $A C\left(W^{\prime}\right)$ with $A C\left(W^{\prime *} \backslash E^{\prime}, W^{\prime *}\right)$.

Let $p \in W \cup \beta$. Consider the mapping

$$
\hat{p}: g \mapsto g^{*}(p), \quad g \in A C(W) ;
$$

it is an algebra homomorphism of $A C(W)$ onto $C$. Hence $\hat{p} \circ T$ is an algebra homomorphism of $A C\left(W^{\prime *} \backslash E^{\prime}, W^{\prime *}\right)$ onto $C$. By [2, Theorem 5.3] there is a point $\sigma^{*}(p) \in W^{\prime *}$ such that

$$
\hat{p} \circ T=f \mapsto f\left(\sigma^{*}(p)\right), \quad f \in A C\left(W^{\prime *} \backslash E^{\prime}, W^{\prime *}\right) .
$$

Thus we have established a mapping $\sigma^{*}$ of $W \cup \beta$ into $W^{* *}$ such that $T f=f \circ \sigma^{*}$ for each $f \in A C\left(W^{\prime *} \backslash E^{\prime}, W^{\prime *}\right)$. Since $C \subsetneq T\left(A C\left(W^{\prime}\right)\right)$, $\sigma^{*}$ must be nonconstant.

To prove the continuity of $\sigma^{*}$, assume that $p_{n} \rightarrow p$ on $W \cup \beta$ but $\sigma^{*}\left(p_{n}\right) \rightarrow q \neq$ $\sigma^{*}(p)$ (note that both $W \cup \beta$ and $W^{\prime *}$ are second-countable). Since $A C\left(W^{\prime *} \backslash E^{\prime}, W^{\prime *}\right)$ separates the points of $W^{\prime *}([2$, Theorem 5.4]), we can find a function $f$ in $A C\left(W^{\prime *} \backslash E^{\prime}, W^{\prime *}\right)$ such that $f\left(\sigma^{*}(p)\right)=0$ and $f(q)=1$. Then $\operatorname{Tf}\left(p_{n}\right) \rightarrow T f(p)=0$ while $f\left(\sigma^{*}\left(p_{n}\right)\right) \rightarrow f(q)=1$. This contradicts the relation $T f=f \circ \sigma^{*}$.

Next we show that $\sigma=\sigma^{*} \mid W$ is analytic. So let $p$ be a point of $W$. Since $\beta^{\prime}$ does not contain removable elements, $U \cap E^{\prime}=\emptyset$ or $U \cap E^{\prime}$ fails to be of class $N_{C}^{\prime}$ in $W^{\prime *}$ for each open set $U \subset W^{\prime *}$ with $\partial U \cap E^{\prime}=\emptyset$ (Theorem 2). Now pick 
out a point $p^{\prime} \in E^{\prime}, p^{\prime} \neq \sigma(p)$, and choose a neighborhood $U_{p^{\prime}}$ of $p^{\prime}$ such that $\sigma(p) \notin \bar{U}_{p^{\prime}}$ and $\partial U_{p^{\prime}} \cap E^{\prime}=\emptyset$. By Lemma 3,AC( $\left.W^{\prime *} \backslash\left(E^{\prime} \cap U_{p^{\prime}}\right), W^{\prime *}\right)$ contains a nonconstant function. Accordingly, we have a nonconstant function $f$ in $A C\left(W^{*} \backslash E^{\prime}, W^{*}\right)$ which is analytic in a neighborhood of $\sigma(p)$. It is elementary that we can find a neighborhood $U_{\sigma(p)}$ of $\sigma(p)$ such that $f$ maps $U_{\sigma(p)} \backslash\{\sigma(p)\}$ smoothly and properly on $f\left(U_{\sigma(p)}\right) \backslash\{f(\sigma(p))\}$. By continuity, there is a neighborhood $U_{p} \subset W$ of $p$ such that $\sigma\left(U_{p}\right) \subset U_{\sigma(p)}$. Since $T f \mid U_{p}$ is analytic, the assertion now follows readily from the relation. $T f \mid U_{p}=f \circ\left(\sigma \mid U_{p}\right)$.

In order to prove the inclusion $\sigma(W) \subset W^{\prime *} \backslash E^{\prime}$, we assume this is not the case, i.e. that $\sigma(p) \in E^{\prime}$ for some $p \in W$. Since $\sigma$ is analytic, we can find a neighborhood $U_{p} \subset W$ of $p$ such that $\sigma$ maps $U_{p}$ properly on $\sigma\left(U_{p}\right)$ and $\partial \sigma\left(U_{p}\right) \cap E^{\prime}=\emptyset$. By Theorem 2, $\sigma\left(U_{p}\right) \cap E^{\prime}$ fails to be of class $N_{C}^{\prime}$. So there is a nonconstant function $f$ in $A C\left(W^{*} \backslash\left(\sigma\left(U_{p}\right) \cap E^{\prime}\right), W^{*}\right)$ (Lemma 3), and, by Lemma 2, $f\left(\sigma\left(U_{p}\right) \cap E^{\prime}\right)$ has interior points. But $\sigma^{-1}\left(E^{\prime}\right) \cap U_{p}$ is compact and totally disconnected, and the same is true of $T f\left(\sigma^{-1}\left(E^{\prime}\right) \cap U_{p}\right)$. Thus we are led to a contradiction.

Altogether, $\psi=\varphi^{\prime-1} \circ \sigma$ is a nonconstant analytic mapping $W \rightarrow W^{\prime}$, admitting a continuous extension $\psi^{*}=\varphi^{\prime *-1} \circ \sigma^{*}: W \cup \beta \rightarrow W^{\prime} \cup \beta^{\prime}$, such that $T f=f \circ \psi$ for every $f \in A C\left(W^{\prime}\right)$. The uniqueness of $\psi$ follows immediately from the fact that $A C\left(W^{\prime}\right)$ separates the points of $W^{\prime}$.

Finally, suppose that also $W$ satisfies the conditions imposed in the theorem on $W^{\prime}$. Let $T$ be an algebra isomorphism of $A C\left(W^{\prime}\right)$ onto $A C(W)$. Let $\psi$ and $\psi^{\prime}$ be the analytic mappings associated with $T$ and $T^{-1}$, respectively. Then $\psi^{\prime} \circ \psi$ and $\psi \circ \psi^{\prime}$ are analytic mappings of $W$ and $W^{\prime}$, respectively, onto themselves which induce the identity homomorphisms of $A C(W)$ and $A C\left(W^{\prime}\right)$, respectively. By uniqueness, $\psi^{\prime} \circ \psi$ and $\psi \circ \psi^{\prime}$ must be the identity mappings on $W$ and $W^{\prime}$. Thus $\psi^{\prime}=\psi^{-1}$, and $\psi$ is a conformal homeomorphism of $W$ onto $W^{\prime}$. The proof is complete.

Corollary. The homomorphism $T$ of the preceding theorem is injective.

- Remarks. (1) The above proof follows closely that of [9, Theorem 1].

(2) As is also obvious from the proof, it follows from Theorem 1 and [2, Theorem 5.3] that the maximal ideal space of $A C\left(W^{\prime}\right)$ is identical with $W^{\prime} \cup \beta^{\prime}$ in case $A C\left(W^{\prime}\right)$ is nontrivial.

(3) In case the range of $T$ is one-dimensional, i.e. $T$ is a homomorphism of $A C\left(W^{\prime}\right)$ onto $C, T$ is again, true, induced by a (point-)mapping $\psi: W \cup \beta \rightarrow W^{\prime} \cup \beta^{\prime}$ ([2, Theorem 5.3]), but it may of course happen that $\psi(W) \in \beta^{\prime}$.

(4) It is clear that in the above characterization of conformal equivalence we cannot dispense with the irremovability of boundary elements. Indeed, let $W$ be a Riemann surface satisfying the hypotheses of Theorem 3 , and let $p \in W$. Then $A C(W \backslash\{p\})$ is identical with $A C(W)$, while $W \backslash\{p\}$ fails, in general, to be conformally equivalent to $W$. 
Example. We will construct a Myrberg-type surface in order to illustrate the situation in the case of infinite genus. Let $E$ be a compact totally disconnected set in the unit disc $\{z \in C|| z \mid<1\}$ failing to be of class $N_{C}^{\prime}$, and let $W$ denote a twosheeted covering of $C \backslash E$ branched over the points $n, n=1,2, \ldots$. Denote by $\varphi$ the projection of $W$ onto $C \backslash E$. It can be shown, by a well-known argument, that each function $f$ in $A C(W)$ admits a representation $f=g \circ \varphi$, where $g$ is an analytic function on $\boldsymbol{C} \backslash E$. Moreover, $g$ is readily seen to be of class $A C(\boldsymbol{C} \backslash E)$. Conversely, every function on $W$ of the form $g \circ \varphi, g \in A C(C \backslash E)$, belongs to $A C(W)$, because $\varphi$ admits a continuous extension $\varphi^{*}: W \cup \beta \rightarrow \hat{\boldsymbol{C}}$. Accordingly, $A C(W)$ fails to separate the points of $W$, and $A C(W)$ is isomorphic to $A C(C \backslash E)$. Note that $A C(\boldsymbol{C} \backslash E)$ is nontrivial by Lemma 3 .

\section{On the field property of $M C(W)$}

In this section we give some characterizations of the case that the class $M C(W)$ constitutes a field.

Theorem 4. Let $W$ be an open Riemann surface of finite genus which satisfies the condition (*). Then the following statements are equivalent:

(1) $M C(W)$ is a field.

(2) $A C(W)=C$.

(3) $M C(W)$ is contained in $B V(W)$, the class of constants and of meromorphic functions of bounded valence on $W$.

(4) $f^{*}(\beta) \in N_{C}^{\prime}$ for some nonconstant function $f \in M C(W)$.

(5) Suppose $W^{*}$ and $E$ are as in Theorem 1. Then $E$ is of class $N_{C}^{\prime}$ in $W^{*}$. Moreover, whenever $M C(W)$ is a field, it can be identified with $M\left(W^{*}\right)$, the field of rational functions on $W^{*}$.

Proof. (1) $\Rightarrow(5)$ : By Lemma 3, it suffices to prove that $A C\left(W^{*} \backslash E, W^{*}\right)$ reduces to the class of constants. So let $f \in A C\left(W^{*} \backslash E, W^{*}\right)$ be arbitrary, and pick out a nonconstant function $g$ in $M\left(W^{*}\right)$. Then $g \mid W^{*} \backslash E \in M C\left(W^{*} \backslash E\right)$, because $E$ is totally disconnected. Let $F \subset W^{*}$ denote the discrete set $B_{g} \cup g^{-1}(\infty)\left(B_{g}\right.$ again denotes the set of branch points of $g$ ). Fix $p_{0} \in E \backslash F$ and select a neighborhood $U$ of $p_{0}$ such that $U \cap F=\emptyset, \partial U \cap E=\emptyset$ and $g \mid U$ is injective. Thus we may regard $h=g \mid U$ as a parametric mapping at $p_{0}$. Let $p, p^{\prime} \in U$, and denote $z=h(p), z^{\prime}=h\left(p^{\prime}\right)$. Since

and, by assumption,

$$
\frac{f\left(h^{-1}(z)\right)-f\left(h^{-1}\left(z^{\prime}\right)\right)}{z-z^{\prime}}=\frac{f(p)-f\left(p^{\prime}\right)}{g(p)-g\left(p^{\prime}\right)}
$$

$$
\lim _{p \rightarrow p^{\prime}} \frac{f(p)-f\left(p^{\prime}\right)}{g(p)-g\left(p^{\prime}\right)} \quad \text { exists (finite or infinite), }
$$


$f \circ h^{-1}$ has a complex derivative (finite or infinite) at every point of $h(U)$. We conclude by the generalized Cauchy-Goursat theorem (see [13, p. 67]) that $f$ is analytic on $U$. By the discreteness of $F, f$ is analytic all over $W^{*}$ and hence constant. The implication follows.

$(5) \Leftrightarrow(4)$ : By Lemma $1, f(E)$ is of class $N_{C}^{\prime}$ whenever $E$ is of class $N_{C}^{\prime}$ in $W^{*}$ and $f \in M\left(W^{*}\right)$. Hence we have $(5) \Rightarrow(4)$. The converse implication is proved in the same way as the implication $(2) \Rightarrow(3)$ in Theorem 2.

$(5) \Rightarrow(3)$ : This follows immediately from the fact that $M C(W)$ can be identified, via the mapping $\varphi^{*}$ (see Theorem 2), with $M\left(W^{*}\right)$.

$(3) \Rightarrow(2)$ : Suppose that $A C(W)$ contains a nonconstant function $f$. By Lemma 2, $f$ cannot be in $B V(W)$.

$(2) \Rightarrow(1)$ : By the remark following Theorem 2, $A C(W)=C$ only if $E \subset W^{*}$ is of class $N_{C}^{\prime}$. Thus $M C(W)$ can be identified with $M\left(W^{*}\right)$, the field of rational functions on $W^{*}$.

Remark. The implications $(4) \Rightarrow(1),(4) \Rightarrow(2)$ and $(4) \Rightarrow(3)$ hold for arbitrary Riemann surfaces as was shown in [5, Theorem 6].

Corollary. Let $W$ be an open Riemann surface of finite genus. Suppose that $M C(W)$ constitutes a field containing a nonconstant function. Then either

(a) every nonconstant function in $M C(W)$ has bounded valence, or

(b) no function in $M C(W)$ has bounded valence.

Remark. We do not know whether the latter alternative can really occur.

\section{References}

[1] Ahlfors, L., and A. Beurling: Conformal invariants and function-theoretic null-sets. Acta Math. 83, 1950, 101-129.

[2] Arens, R.: The closed maximal ideals of algebras of functions holomorphic on a Riemann surface. - Rend. Circ. Mat. Palermo (2) 7, 1958, 245-260.

[5] Gamelin, T. W.: Uniform algebras. - Prentice-Hall, Inc., Englewood Cliffs, N. J., 1969.

[4] Garnetr, J.: Analytic capacity and measure. - Lecture Notes in Mathematics 297, SpringerVerlag, Berlin-Heidelberg-New York, 1972.

[5] JÄrvi, P.: Meromorphic functions on certain Riemann surfaces with small boundary. - Ann. Acad. Sci. Fenn. Ser. A I 5, 1980, 301-315.

[6] Jurchescu, M.: A maximal Riemann surface. - Nagoya Math. J. 20, 1962, 91-93.

[7] RaO, K. V. R.: Imbedding closed Riemann surfaces in $C^{n}$. - Proc. Amer. Math. Soc. 19, 1968, $30-32$.

[8] Richards, I.: On the classification of noncompact surfaces. - Trans. Amer. Math. Soc. 106, 1963, 259-269.

[9] Royden, H. L.: Rings of analytic and meromorphic functions. - Trans. Amer. Math. Soc. 89, 1956, 269-276.

[10] Sario, L., and K. OiKawa: Capacity functions. - Die Grundlehren der mathematischen Wissenschaften 149, Springer-Verlag, Berlin-Heidelberg-New York, 1969. 
[11] StoïLow, S.: Leçons sur les principes topologiques de la théorie des fonctions analytiques. Gauthier-Villars, Paris, 1956.

[12] Titus, C. J., and G. S. Young: The extension of interiority, with some applications. - Trans. Amer. Math. Soc. 103, 1962, 329-340.

[13] Trokнiмchuk, Yu. Yu.: Continuous mappings and conditions of monogeneity. - Israel Program for Scientific Translations, Jerusalem, 1964.

\author{
University of Helsinki \\ Department of Mathematics \\ SF-00100 Helsinki 10 \\ Finland
}

Received 20 September 1979

Revision received 23 January 1980 